\title{
Methotrexate normalizes folate pathway genes in RA
}

$\mathrm{F}$

or decades, methotrexate has

formed the foundation of therapy in

RA, either alone or in combination

with other drugs; however, its mechanisms of action remain poorly understood, and many patients experience a lack of clinical response or adverse events. In a study now published in Arthritis \& Rheumatism, Blits et al. identify dynamic alterations in the folate pathway that occur in RA and in response to methotrexate treatment. Their findings open the door to future studies on the biochemical processes that underlie the mechanism of action and also the toxicity of methotrexate.

Blits et al. examined the expression levels of genes encoding components of the folate pathway in peripheral blood samples from methotrexate-treated and methotrexatenaive patients with RA, as well as healthy individuals. Gene expression microarray analysis was performed in a test cohort comprising 10 untreated patients with RA, 25 methotrexate-treated patients with RA and 15 healthy individuals. The microarrayderived data for 17 folate pathway genes were subsequently validated by real-time PCR (RT-PCR) in an independent cohort of 28 methotrexate-naive patients with RA, 151 methotrexate-treated patients with RA and 24 healthy persons.

"This methodology was successfully applied to identify gene expression signatures and relevant pathways involved in the pathophysiology of RA and in relation to methotrexate treatment," explains Gerrit Jansen, (from the VU University Medical Center, Netherlands), who contributed to the study. "The most striking finding was that, relative to healthy individuals, a group of treatment-naive patients with RA demonstrated a significant upregulation of basal folate metabolism, represented by increased expression of several genes encoding folate metabolizing enzymes and folate/methotrexate efflux transporters, concurrent with an immune-activation signature," continues Jansen.

Interestingly, "in the RA patient group receiving methotrexate treatment, the upregulated genes were largely normalized in their expression to levels detected in healthy individuals," says Jansen. Although differences in disease duration and bone erosions between the methotrexatenaive and methotrexate-treated groups could have confounded the results, subanalyses suggested this was not the case; furthermore, neither potential changes in immune-cell subsets with methotrexate treatment, nor the duration of treatment seemed to explain the differences in folate gene expression profiles observed.

Together, these findings suggest that, in untreated patients with RA, increased demand for folate-dependent de novo purine and pyrimidine biosynthesis, driven by activation of the immune system, could cause a concomitant upregulation of the folate pathway. Subsequently, treatment with methotrexate might reduce the demand for folate, whether directly by interfering with folate metabolism or due to suppression of inflammation via other mechanisms, thus restoring folate homeostasis. "This study is the first of its kind to provide information on the dynamic features of folate metabolism and homeostasis in blood cells from patients with RA and the influence of methotrexate treatment on this pathway in RA," claims Jansen.

Jansen suggested that, as the current study had a cross-sectional design, longitudinal analysis during the course of methotrexate treatment in patients with RA could be relevant in determining whether folate pathway gene expression has value in predicting responsiveness to methotrexate. In addition, "the novel folate pathway gene signatures under inflammatory conditions have potential for experimental therapeutic intervention using folate antagonists that inhibit the targets identified; in an anticancer setting, these novel generation antifolates are already employed to overcome methotrexate resistance," notes Jansen.

Bruce Cronstein (NYU School of Medicine, USA), who was not involved in the study, feels more information is needed

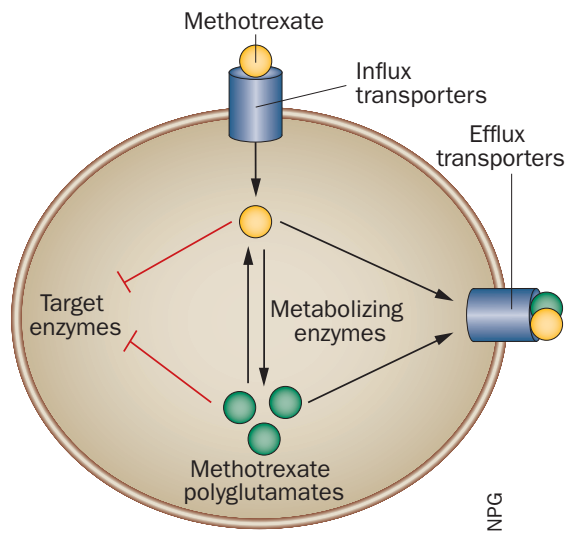

before the implications of these findings can be fully understood. "Overall the approach taken was appropriate and the results appear to be sound; however, the actual expression of the enzymes in question should be tested at some point," says Cronstein. "Furthermore, the authors did not examine cells from patients with another chronic inflammatory disease to determine whether the increased expression of the enzymes observed in patients with RA is not simply a reflection of inflammation (and inflammatory stimulation of the peripheral blood cells) and the reduction in expression reflects a general reduction in inflammation and cellular stimulation." Thus, "it would be interesting to determine whether any of the postulated mechanisms of action of methotrexate mediate the change in enzyme expression in cultured cells exposed to inflammatory stimuli," he opines. "Another question raised by this study is the effect of folate itself on the message for these enzymes, as many patients with RA are taking folic acid or even folinic acid," concludes Cronstein.

\section{David Killock}

